\title{
Patterned grafting of polymer brushes onto flexible polymer substrates
}

\author{
Celestino Padeste ${ }^{\text {a) }}$ and Harun H. Solak \\ Laboratory for Micro- and Nanotechnology, Paul Scherrer Institut, 5232 Villigen PSI, Switzerland
}

Hans-Peter Brack, ${ }^{\text {b) }}$ Michal Slaski, Selmiye Alkan Gürsel, and Günther G. Scherer
Electrochemistry Laboratory, Paul Scherrer Institut, 5232 Villigen PSI, Switzerland

(Received 2 June 2004; accepted 17 August 2004; published 10 December 2004)

\begin{abstract}
Selective exposure of polymer substrates to extreme ultraviolet (EUV) radiation followed by graft polymerization of a second polymer is used to create patterns of polymer brushes. A key feature of this additive structuring process is the possibility to combine the properties of a polymer substrate in precisely predefined regions with the properties of the grafted material. Styrene brushes were grafted onto poly(ethylene-alt-tetrafluoroethylene) (ETFE) substrates after exposure to synchrotron EUV radiation in an interference lithographic setup. The grafted chains are covalently bound to the surface, which allows physical or chemical post-processing without deterioration of the grafted structures. () 2004 American Vacuum Society. [DOI: 10.1116/1.1805542]
\end{abstract}

\section{INTRODUCTION}

Polymer brushes are polymer chains tethered at one end to a substrate surface with high enough chain density so that the chains mainly extend away from the surface resembling a brush formation. These materials attract interest because they form strongly adherent films and their properties can be tailored by the brush composition and structure. In particular desired support materials with specific properties such as mechanical strength or thermal stability can be endowed with such surface characteristics as wetting properties or specific binding properties originating from the grafted material.

The brushes are usually grown through polymerization of reactive monomers starting at reactive sites in the support material. These sites are often created by binding or deposition of specific starter molecules or precursors thereof which are then activated in a subsequent step. ${ }^{1-4}$

The spatially resolved activation or deactivation of such reactive sites has been demonstrated as a means to create patterns of polymer brushes on specially prepared surfaces. On gold or silicon surfaces, self-assembled monolayers (SAMs) of precursor molecules were first deposited, followed by chemical derivatization to give the surface-bound initiators for the subsequent grafting reaction. Using focused electron beams for selective deactivation of the initiators, grafting of structures with a lateral resolution in the $50 \mathrm{~nm}$ range has been demonstrated. ${ }^{2,3}$ Due to the chemical amplification through the grafting reaction, such grafted patterns may find application as high resolution photoresists. ${ }^{4}$ However, this technique has some drawbacks including the limited choice of support materials that allow the formation of SAMs, and the potentially weak adhesion of the grafted material, which relies on the interaction between the SAMforming molecules and the base material.

Recently we reported on first experiments of the adaptation of well-known radiation grafting processes to create

\footnotetext{
${ }^{a)}$ Electronic mail: celestino.padeste@psi.ch

${ }^{b)}$ Present address: Swiss Federal Institute of Technology, Department of Industrial Management and Production Technology, CH-8006 Zürich.
}

micro- and nano-patterns of polymer brushes on polymeric substrates. ${ }^{5}$ In conventional radiation grafting a base polymer is irradiated with high energy radiation, e.g., gamma-rays or $\mathrm{MeV}$ electrons, in order to create radicals serving as the initiators for the grafting of the second polymer. In such processes the density of grafted chains is mainly controlled by the irradiation dose, while the reaction conditions such as concentration, time and temperature influence the length of the grafted polymer chains. ${ }^{6}$

In this work we focus on the use of extreme ultraviolet (EUV) light in a synchrotron-based interference setup ${ }^{7}$ to create the initiator radicals in periodic line-space or dot arrays (see Fig. 1). The radicals are created in a limited depth range of about a few tenths of a micrometer near the surface because of the high absorption of EUV light by the substrate. Compared to exposure with electrons no limitation of the process due to the charging of the nonconducting polymer sample is expected. The grafting reaction carried out with the exposed samples should be limited to the parts of the surface where radicals had been created.

The surface micro- and nano-grafting process is clearly different from the conventional lithographic approach in several ways. Most resists used in photolithography are tuned to show a strongly nonlinear dose-sensitivity relation. In this way, a binary process is achieved in that the resist after irradiation and development is either completely dissolved or solvent-stable. This is a prerequisite for the usually carried out subsequent pattern transfer processes. In contrast, the number of surface bound polymer chains obtained in a grafting process depends on the irradiation dose over a wide range. This leads to the ability to gradually change the surface density of the graft material by varying the dose. Provided that the growth conditions are maintained in the socalled brush-regime, (which is characterized by the linear dependence of the thickness on the number of grafted chains per surface area) dose variation can be used as a means to achieve different grafting heights. As an "additive process," the micro- and nano-grafting works without any sacrificial layers, unlike the way photoresist are used in conventional 

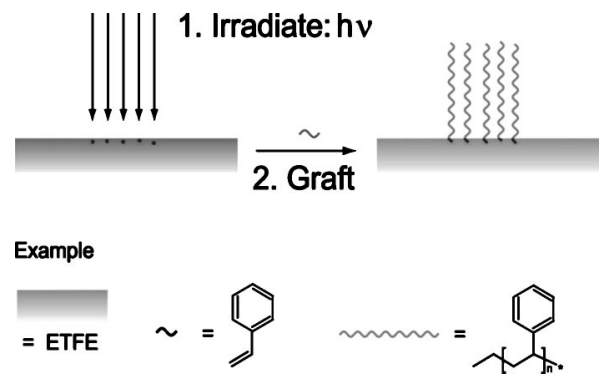

FIG. 1. Scheme of the process. Selective irradiation of an ETFE-Foil with EUV radiation creates radicals in the polymer. Subsequent grafting of polystyrene leads to the growth of a three-dimensional structure.

photolithography in most cases. During the process the grafting material with selected properties directly forms the intended structures. In addition, grafted polymer patterns can be subsequently modified with physical or chemical processes, such as thermal annealing or chemical functionalization without losing the pattern definition thanks to the covalent bonding of the pattern to the substrate surface.

It is the aim of this article to explore some of these unique features of the micro- and nano-grafting process for the system of polystyrene grafted onto commercial ETFE films.

\section{EXPERIMENT}

ETFE-substrates: Extruded Nowoflon ET-6235 films having thicknesses of $100 \mu \mathrm{m}$ were purchased from Nowofol $\mathrm{GmbH}$, Siegsdorf, Germany. The average molar weight of the Dyneon ET-6235 copolymer used to make these films is approximately 400000 Dalton. ${ }^{8}$ Typical physical properties of these ETFE films such as their crystallinity, glass transition temperature, melt flow index, and orientation have been reported in detail. ${ }^{9}$ To obtain a flat test surface, a piece of ETFE film was placed between two polished 4" silicon wafers. In a hot press which is optimized for nano-imprint lithography, this sandwich was heated for $5 \mathrm{~min}$ at $230^{\circ} \mathrm{C}$ under a pressure of $200 \mathrm{~N} / \mathrm{cm}^{2}$. The procedure results in a reduction of film thickness of about 5\%-10\%, and a drastic reduction in surface roughness. No evidence of thermal degradation of the polymer was observed due to this treatment.

EUV exposures were done in vacuum $\left(<10^{-5} \mathrm{mbar}\right)$ at the "x-ray interference lithography" beamline of the Swiss Light Source. The beamline uses undulator light with a central wavelength of $13.4 \mathrm{~nm}(92 \mathrm{eV})$ and $\sim 4 \%$ spectral bandwidth. The incident EUV power on the sample was several $\mathrm{mW} / \mathrm{cm}^{2}$ and the delivered dose was controlled in the range of $2-20 \mathrm{~mJ} / \mathrm{cm}^{2}$ using a fast beam shutter. To define the exposed areas on the sample a TEM-grid with features in the range of $\geqslant 10 \mu \mathrm{m}$ was used as shadow mask in proximity to the sample. Sample irradiation by means of $x$-ray interference lithography was carried out according to the methods described in our earlier publication. ${ }^{7}$ Silicon nitride masks with gratings of various periods were used to create interference patterns with periods in the range of $100-1000 \mathrm{~nm}$. The irradiated samples were stored in air in a deep freezer $\left(-80{ }^{\circ} \mathrm{C}\right)$ until further processing.

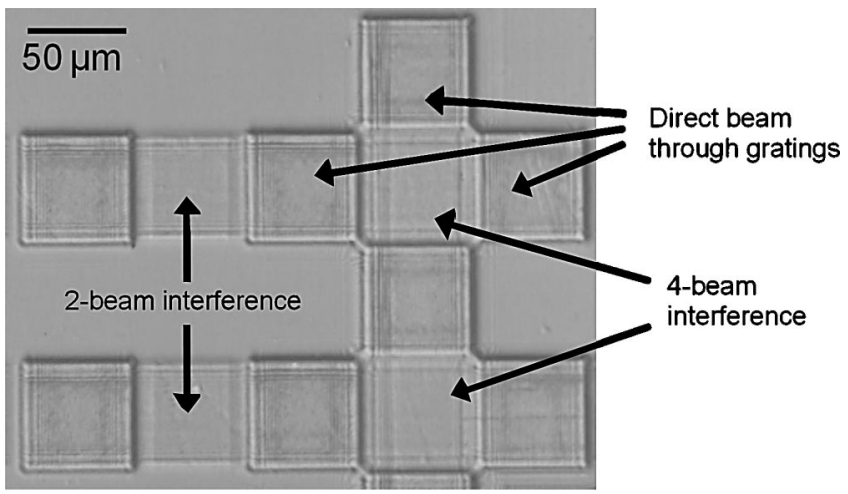

FIG. 2. Optical micrograph of surface structures produced with the micro-/ nano-grafting technique.

Sample grafting was carried out under inert atmosphere in glass reactor tubes placed in a water bath preheated to the desired reaction temperature. The monomer solution contained 5\% to 20\% (v/v) of styrene (Fluka, p.a.) in an 11:5 mixture $(\mathrm{v} / \mathrm{v})$ of isopropanol and water. When the reaction time was over, the grafted samples were thoroughly washed with isopropanol. Reaction temperature was $60^{\circ} \mathrm{C}$, and reaction times of 15 to $30 \mathrm{~min}$ were evaluated in this study.

Atomic force microscopy (AFM). A Digital Instrument Nanoscope III/Dimension 3100 was used in the tapping mode. Samples were mounted on pieces of silicon wafers and sputter-coated with a few nanometer thin Au film to reduce electrical charging during the measurements.

\section{RESULTS AND DISCUSSION}

\section{A. Process characteristics}

The process to generate micro- and nanopatterns of grafted polymer brushes is schematically depicted in Fig. 1. From the enormous variety of polymers that could undergo such reactions, we selected the grafting of polystyrene onto ETFE films, a process which was well known in-house from the bulk grafting of ETFE for fuel cell applications. ${ }^{9}$ Irradiation sequences with varying EUV dose were carried out on individual samples, which were then grafted at different reaction conditions, in order to elucidate the critical parameters for the process.

Figure 2 shows an optical micrograph of a sample exposed in an interference setting after grafting. A large dynamic range of the process becomes evident in this picture. The thickness of the grafted material is highest in the regions where the high-intensity direct EUV beam was incident on the surface. The interference patterns cannot be resolved with visible light microscopy but grafting in this region, in which the applied dose is lower by at least one order of magnitude, is still visible. Moreover, the Fresnel intensity fringes near the edges of the exposed squares are clearly apparent in areas exposed by both direct and diffracted beams.

A more quantitative assessment of the dynamic range of the process is given in Fig. 3. The grafting height of fully exposed areas was determined as a function of irradiation 


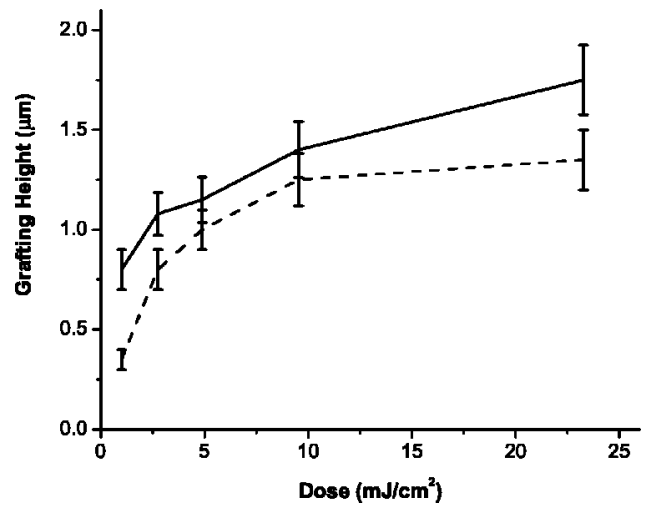

FIG. 3. Dose dependence of the thickness of polystyrene patterns grafted in a $20 \%$ styrene solution for $30 \mathrm{~min}$, before (straight line) and after (dashed line) treatment with toluene.

dose and reaction conditions. The increasing height of the structures with increasing dose is an effect usually assigned to the brush-regime. In this range, the grafted chains are packed in a way, that they mainly extend perpendicular to the surface. As the dose increases, the number of reactive sites and thus the number of grafted polymer chains increase. This forces the chains to assume more extended conformations in the perpendicular direction, leading to a thicker grafted film.

Figure 4(a) shows a grafted micrograting. In this structure the height profile reflects the intensity profile of the interfering EUV-beams hitting the surface. The high dynamic range of the technique will allow its use for the creation of more complex surface topologies. Features irradiated on one surface with different dose show up in different height after the grafting process. This effect can be used to produce surface relief structures.

\section{B. High resolution limitation}

At the grafting conditions used in this study, a film thickness of $100 \mathrm{~nm}$ to $1.5 \mu \mathrm{m}$ was observed at the highest irritation dose. If the chains were standing upright on the sur- face, then the thickness would correspond to the chain length. In reality the chains must be considerably longer singe they are coiled and not all standing upright. This is evident in a higher-resolution image of grafted structures [Fig. 4(b)]. Hair-like features in the image are interpreted as bundles of polymer chains extending in all different directions at the surface of the grafted structures. The length of the chains is an important parameter that may have an impact on the spatial resolution.

The quality of the grafted features could be improved by increasing the grafting density, and by limiting the chain lengths smaller than the desired feature size. Alternatively the conformations of the grafted brushes could be modified with post-processing steps taking advantage of the covalent bonding to the substrate as we demonstrate in an example below.

\section{Post-processing}

Due to the covalent nature of the bonding to the base polymer, post-processing of the grafted chains, such as thermal treatment, partial dissolution or chemical derivatization is possible without losing the grafted material from the surface.

Toluene is a good solvent for polystyrenes and is frequently used to remove unreacted monomer from the grafted material. As shown in Fig. 3, toluene treatment leads to a reduction in thickness of the grafted polystyrene layers, which can be assigned to a re-ordering of the grafted chains to result in a more densely packed polymer. AFM images of nanostructures with an average grafting height below $20 \mathrm{~nm}$ confirm this interpretation (Fig. 5). Instead of the hairy structures as described above, little droplets of polystyrene are found after toluene treatment, which may be explained as follows: It needs to be noted that the ETFE-polymer exhibits a very low surface energy. Therefore, the forces between the polystyrene-toluene solution and the surface are very low, leading to the formation of droplets in the later stages of evaporation of the solvent. These droplets tend to form in the
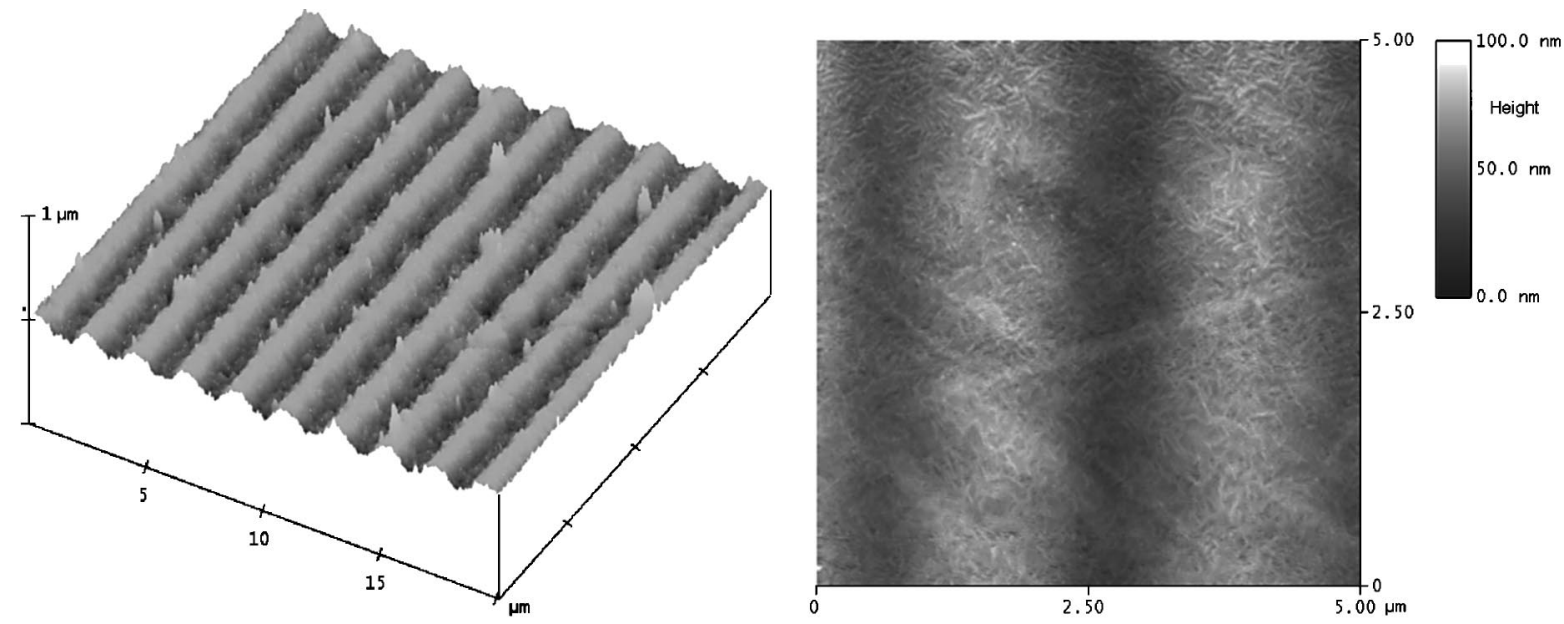

FIG. 4. (a) Thickness modulation in a $2 \mu \mathrm{m}$ period grid pattern produced with interference lithography grafted at high concentration. (b) High resolution image of the same structure. 

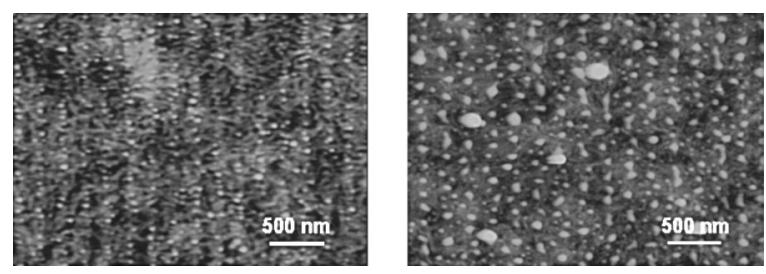

FIG. 5. $200 \mathrm{~nm}$ period pattern (low dose and grafted at low concentration) before and after treatment in toluene.

places with the highest density of grafted polymer chains. After total solvent evaporation polystyrene formed in the shape of the droplets remains. When applying this partial dissolution process to structures grafted at higher levels, a significant improvement of the structure definition was observed and well resolved $200 \mathrm{~nm}$ period structures were obtained (Fig. 6).

The covalent nature of the bonding between the base polymer and the grafted material allows a post-processing of the grafted polymer without losing the formed structures. For the grafted polystyrene there are many chemical derivatization possibilities since the aromatic ring can undergo various specific chemical reactions.
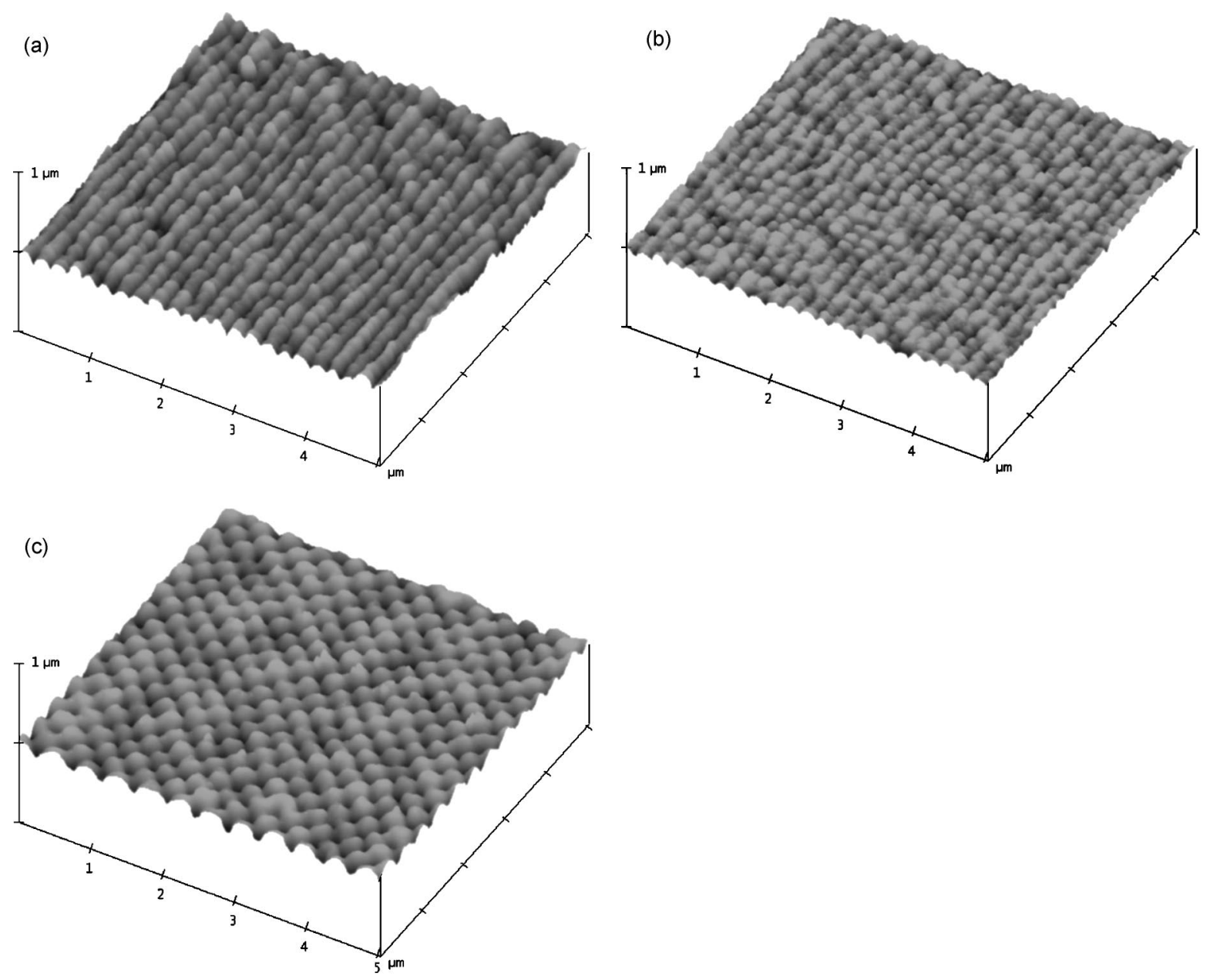

\section{CONCLUSIONS}

The micro- and nano-grafting process represents in many ways an alternative to conventional lithographic structuring processes. As an additive process which sensitively reacts to irradiation dose variations, it allows to grow complex surface topologies in one chemical reaction step by choosing suitable beam intensity distributions. The uniformity of the grafted structures is not yet satisfying for possible applications but this may be improved by better control of the grafting conditions and by adding crosslinkers, which interlink the grafted chains leading also to improved mechanical strength.

In our first experiments, we did not focus on the different properties of the base polymer and the grafted material. However, the experiments with toluene as the solvent indicate that the grafted chains are firmly bound to the ETFE substrate. This stability of the chemical anchoring is a prerequisite to chemically modify the grafted chains in order to obtain specific biochemical or chemical functionality. Together with all the variety of radically induced grafting reactions this opens a very wide range of achievable properties of the grafted regions, and thus to many different applications, e.g., for smart materials and in biosciences.

FIG. 6. $200 \mathrm{~nm}$ period structures created with (a) 2 beam and [(b) and (c)] 4 beam interference, respectively. Grafted with $20 \%$ styrene, 15 min, $60{ }^{\circ} \mathrm{C}$; sample treated in toluene. 


\section{ACKNOWLEDGMENT}

EUV interference exposures were performed at the XILbeamline at the Swiss Light Source, Paul Scherrer Institut, Villigen, Switzerland.

${ }^{1}$ M. Freemantle, C \& EN, April 14, 41 (2003).

${ }^{2}$ C. L. Henderson, S. Barstow, A. Jeyakumar, K. McCoy, D. W. Hess, and L. M. Tolbert, Mater. Res. Soc. Symp. Proc. 705, 3 (2002).

${ }^{3}$ U. Schmelmer, R. Jordan, W. Geyer, W. Eck, A. Gölzhäuser, M. Grunze, and A. Ulman, Angew. Chem., Int. Ed. 42, 559 (2003).
${ }^{4}$ I. S. Maeng and J. W. Park, Langmuir 19, 4519 (2003).

${ }^{5}$ H.-P. Brack, C. Padeste, M. Slaski, S. Alkan, and H. H. Solak, J. Am. Chem. Soc. 126, 1004 (2004).

${ }^{6}$ T. Kawai, K. Saito, and W. Lee, J. Chromatogr., B: Biomed. Appl. 790, 131 (2003).

${ }^{7}$ H. Solak, C. David, J. Gobrecht, V. Golovkina, F. Cerrina, S. O. Kim, and P. F. Nealey, Microelectron. Eng. 67-68, 56 (2003).

${ }^{8}$ H. Hartl, Dyneon GmbH, Burgkirchen, Germany, personal communication (1998).

${ }^{9}$ H. P. Brack, H. G. Bührer, L. Bonorand, and G. G. Scherer, J. Mater. Chem. 10, 1795 (2000). 